\title{
Epileptiform abnormalities during sleep in Rett syndrome ${ }^{1}$
}

\author{
Michael S. Aldrich *, Elizabeth A. Garofalo ** and Ivo Drury * \\ * Department of Neurology and ${ }^{* *}$ Division of Pediatric Neurology, University of Michigan, Ann Arbor, MI (U.S.A.)
}

(Accepted for publication: 22 September 1989)

\begin{abstract}
Summary We recorded all-night electroencephalograms (EEGs)/polysomnograms on 2 consecutive nights from 4 children (ages 4-11 years) with Rett syndrome. The first $10 \mathrm{sec}$ of each $60 \mathrm{sec}$ epoch were analyzed with counts of left and right hemisphere spikes and correlated with sleep stage. Spike counts were lowest during wakefulness. Spikes were most frequent over parasagittal regions during all sleep stages and varied from $0.28 \pm 0.03$ to $40.4 \pm 0.7$ (mean \pm S.E.M.) spikes/hemisphere/min. Spike counts were 51-109\% higher during NREM sleep than during REM sleep. In 3 of 4 subjects, spikes were most frequent during light NREM sleep. Spikes increased in frequency during the second half of the night. We conclude that in Rett syndrome, epileptiform activity is maximally expressed in stage 1-2 NREM sleep and during the early morning hours. Sleep EEG features may be useful in the diagnosis of Rett syndrome.
\end{abstract}

Key words: Rett syndrome; Epilepsy; EEG; Sleep

Rett syndrome is a degenerative condition in girls characterized by stereotyped hand movements, disordered respiratory patterns and loss of language and motor skills. The electroencephalogram (EEG) is almost always abnormal with a variety of findings, including rhythmic slow wave activity, and diffuse, focal or multifocal spike and sharp waves (Niedermeyer et al. 1986; Garofalo et al. 1988). Daytime EEGs often show a marked increase in epileptiform activity during sleep (Verma et al. 1986; Glaze et al. 1987), but the relationship to sleep stages has not been defined. To better characterize epileptiform abnormalities during sleep in these patients, we performed allnight recordings on 4 children with Rett syndrome.

\footnotetext{
$\overline{1}$ Presented in part at the American Academy of Neurology Annual Meeting, Cincinnati, OH, April 1988.

Correspondence to: Dr. M.S. Aldrich, Department of Neurology, Taubman Center 1920/0316, University of Michigan Medical Center, 1500 East Medical Center Drive, Ann Arbor, MI 48109-0316 (U.S.A.).
}

\section{Subjects and methods}

All children were clinical stage III (pseudo-stationary stage) according to criteria of Hagberg et al. (1986). Case histories are summarized below.

Case no. 1 was a $7.25 \mathrm{lb}$ product of a normal pregnancy. She walked at 15 months but at 24 months she only babbled and did not speak words. At 2.5 years she stopped playing with toys, and by age 3 she had stopped feeding herself and often bit or clapped her hands. A waking EEG was normal.

At age 4 she began having brief generalized seizures characterized by stiffening of the arms and legs, eye deviation to the right and jerking of the extremities. By age 6, seizures occurred up to 50 times/day. Several EEGs between ages 4 and 7 showed background slowing with frequent bisynchronous polyspike-wave complexes with rightsided emphasis. Epileptiform discharges increased in frequency during drowsiness and stage 2 sleep. Brain computed tomography was normal.

On examination at age 6, she was below the fifth percentile for height and weight. She was 
ataxic, inattentive to visual and auditory stimuli, did not speak, and had almost continuous clapping of the hands. She had frequent apneic spells lasting up to $60 \mathrm{sec}$ that were not associated with ictal EEG patterns. Seizures continued despite trials of several anticonvulsants. At age 10, seizures abated and by age 11 she had been free of seizures for 6 months. She remained unable to speak or feed herself.

Case no. 2 was a $6.75 \mathrm{lb}$ product of a normal pregnancy. She crawled at 7 months, walked at 15 months and appeared normal until about 18 months when she lost interest in her surroundings. Intermittent handwashing movements began. At age 2 , she was clumsy and fell frequently. Examination revealed a wide-based gait and diffuse hyperreflexia. An EEG showed excessive intermixed slow waves; during sleep there were spikes and spike-waves. At age 2.5 she had constant handwringing and no useful hand movement. At age 4, episodic hyperventilation and apnea were noted. Brief generalized seizures began, characterized by head dropping, eye closure, and unre- sponsiveness. At age 5 she was profoundly retarded with no speech and was unable to feed herself.

Case no. 3 was an $8.5 \mathrm{lb}$, full-term product of an uncomplicated pregnancy. She sat at 8 months, crawled at 12 months, and walked and spoke a few words at 15 months. At 15 months she became disinterested in her parents, stopped speaking, and developed a wide-based gait. Thorough evaluation for a degenerative disease was unrevealing. At age 2 years, a waking EEG showed a mild excess of slow activity. At age 3, bilateral spikes were noted on EEG and several brief generalized seizures occurred during sleep. Seizures improved with carbamazepine. At age 4.5 she had gait ataxia, constant handwashing movements, and did not speak. An EEG showed frequent spikes over the parasagittal regions which increased in frequency during sleep (Fig. 1).

Case no. 4 was a $5 \mathrm{lb} 11 \mathrm{oz}$ product of pregnancy complicated by phenobarbital treatment of maternal hypertension. At 18 months, she was walking and at 20 months she had a 15-20 word

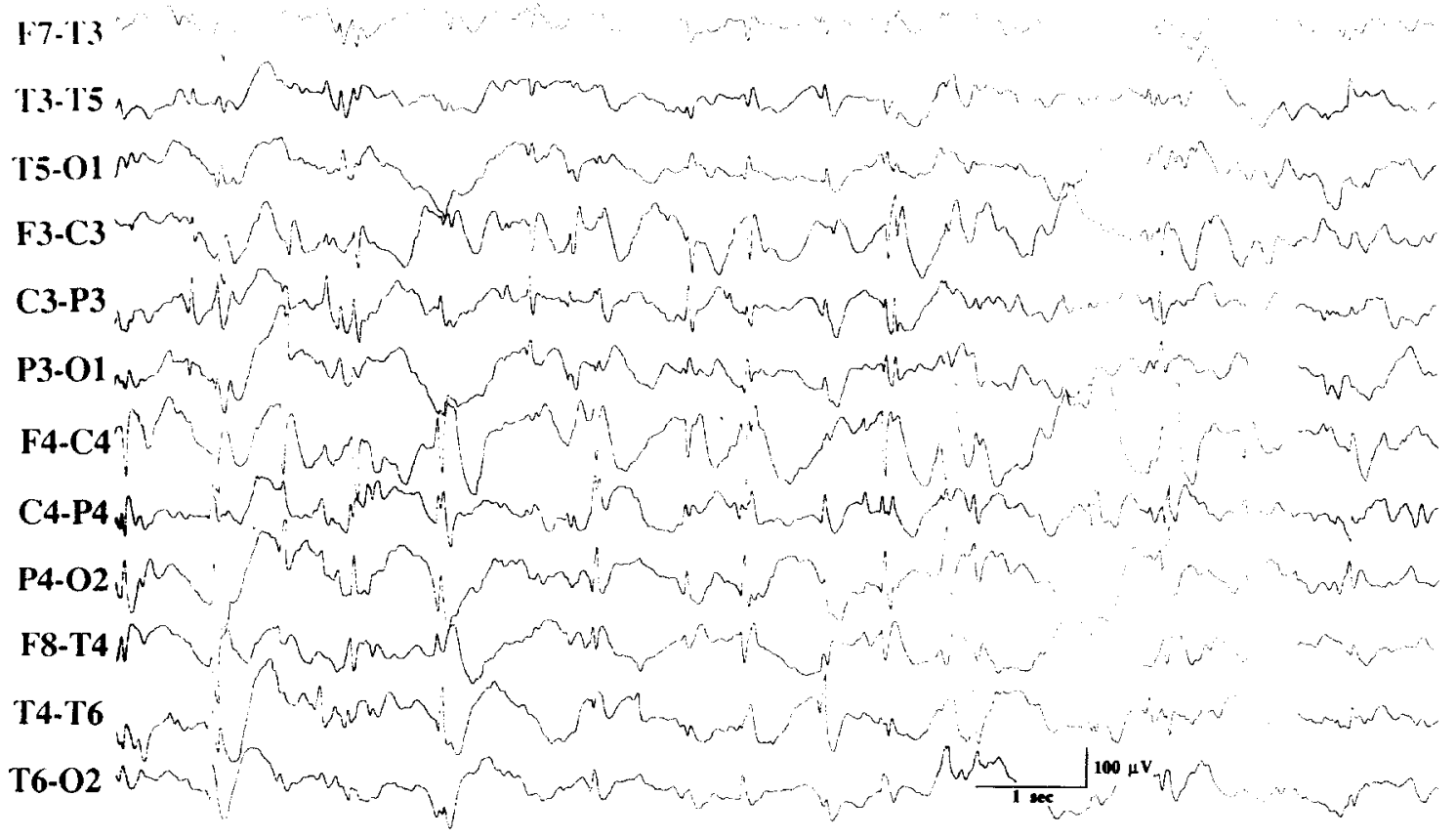

Fig. 1. This EEG tracing from case no. 3 shows a portion of stage 2 NREM sleep. Most spikes are of brief duration (30-60 msec) and are maximal over centroparietal regions with some spread into temporal derivations. A few spikes are maximal over temporal regions and are of slightly longer duration. 
vocabulary. Over the next 9 months, she lost purposeful use of her hands, stopped walking and speech was limited to 2-3 words. An EEG showed left centro-temporal spikes. Generalized seizures began at age 4.5 years and were treated with phenobarbital and phenytoin. At age 7.5 years she was ataxic, unable to walk or speak, and had no purposeful hand use.

After informed parental consent, we recorded all-night EEG/polysomnograms from these 4 children. Because of the potential for a 'first-night effect' that might alter the findings, we recorded the subjects for 2 consecutive nights. Sixteen scalp electrodes were placed using the international 10 20 system. Recordings were made with a 21-channel polygraph (Nihon-Kohden Corp.) at 30 $\mathrm{mm} / \mathrm{sec}$ paper speed using a longitudinal, bipolar montage. Electrocardiogram, respiratory effort, nasal/oral air flow, and pulse oximetry (Biox 3700, Ohmeda Corp.) were also recorded.

Sleep studies were scored for sleep stages using standard criteria (Rechtschaffen and Kales 1968). The number of left and right parasagittal spikes during the first $10 \mathrm{sec}$ of each $60 \mathrm{sec}$ epoch were counted independently by 2 experienced electroencephalographers (I.D. and E.G.). For wakefulness and for each stage of sleep, we determined the mean of the spike counts for the 2 electroen- cephalographers for each epoch and multiplied this value by 6 to obtain the mean number of spikes per hemisphere per minute. Statistical differences were determined using analysis of variance.

\section{Results}

All 4 subjects had slow background EEG during wakefulness. During sleep, in 2 subjects (nos. 2 and 3), K-complexes and sleep spindles were difficult to identify. All subjects had well-defined rapid eye movements and muscle atonia during REM sleep but in 2, slow activity and background amplitude of the EEG were similar during REM and NREM sleep. In 1 subject (no. 4), the amount of REM sleep was reduced. Polysomnographic results are shown in Table I.

All 4 subjects had normal respiration during sleep. All had epileptiform activity during sleep and 3 had epileptiform discharges during wakefulness, but in all subjects spike counts were lower during wakefulness than during sleep. There were no significant differences between 1st and 2nd nights. No seizures occurred.

Spikes per minute of sleep were most prevalent over parasagittal regions during all sleep stages.

\section{TABLE I}

Polysomnographic measures in Rett syndrome.

\begin{tabular}{|c|c|c|c|c|c|}
\hline & \multirow[b]{2}{*}{ Age (years) } & \multicolumn{3}{|c|}{ Patient } & \multirow[t]{2}{*}{ Mean } \\
\hline & & 2 & 3 & 4 & \\
\hline Recording time (min) & 497.5 & 466.4 & 474.8 & 476.2 & 478.7 \\
\hline Total sleep time (min) & 492.0 & 414.1 & 455.3 & 445.8 & 451.8 \\
\hline Latency to sleep (min) & 0.5 & 31.4 & 1.3 & 0.1 & 8.3 \\
\hline Latency to REM sleep (min) & 134.7 & 134.8 & 31.0 & 120.3 & 117.7 \\
\hline Sleep efficiency (\%) & 98.9 & 95.4 & 96.1 & 93.8 & 96.1 \\
\hline Number of arousals & 32.0 & 17.5 & 12.0 & 27.5 & 22.3 \\
\hline Number of awakenings & 7.5 & 15.5 & 13.0 & 33.0 & 17.3 \\
\hline Number of awakenings $>2 \mathrm{~min}$ & 0.5 & 3.0 & 1.5 & 2.5 & 1.9 \\
\hline \% Wake & 1.1 & 11.1 & 4.0 & 6.3 & 5.6 \\
\hline$\%$ stage 1 sleep & 7.8 & 13.3 & 28.9 & 22.1 & 18.0 \\
\hline \% stage 2 sleep & 41.5 & 32.3 & 25.4 & 37.3 & 34.1 \\
\hline$\%$ stage $3-4$ sleep & 34.0 & 21.6 & 20.4 & 27.6 & 25.9 \\
\hline \% REM sleep & 15.3 & 21.4 & 21.1 & 6.5 & 16.1 \\
\hline Minimum oxygen saturation & 92.5 & 94.0 & 91.5 & 92.5 & 92.6 \\
\hline Average oxygen saturation & 97.0 & 96.0 & 96.5 & 96.5 & 96.5 \\
\hline
\end{tabular}




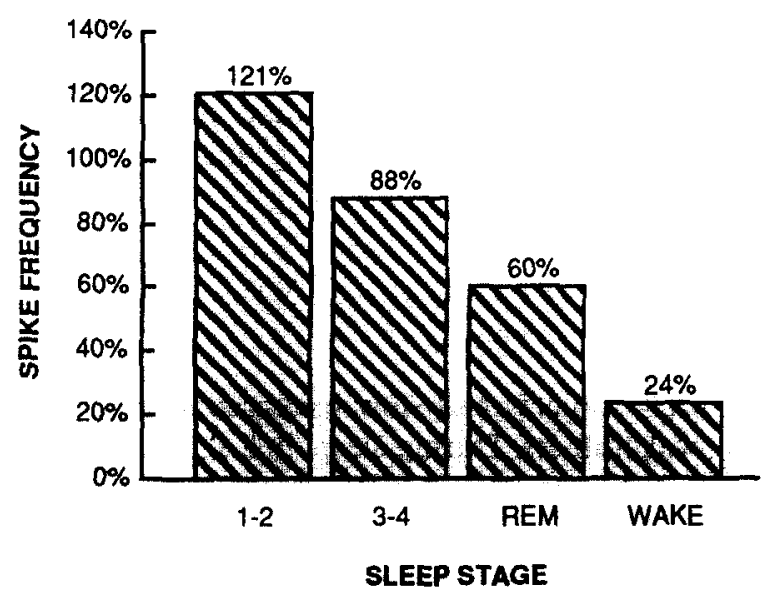

Fig. 2. Spike frequency during wakefulness, REM sleep, light NREM sleep, and deep NREM sleep as a percentage of mean spike frequency during all sleep. The values are the mean of the percentages for the 4 patients. Spike frequency is $80 \%$ higher during NREM sleep than during REM sleep.

Most spikes were of short duration, $30-60 \mathrm{msec}$, had a field restricted to centroparietal derivation, and averaged 50-100 $\mu \mathrm{V}$. Some spikes were 50-150 msec in duration, had amplitudes up to 150-250 $\mu \mathrm{V}$, and had a broader field that included midtemporal and posterior temporal derivations. Occa- sional spikes had a midtemporal or posterior temporal maximum. Morphology, distribution and amplitude of spikes were similar during REM and NREM sleep. There was a wide variation in spike frequency, from $0.28 \pm 0.03$ (mean \pm S.E.M.) spikes/min in subject no. 1 to $40.4 \pm 0.7$ in subject no. 3 .

For each subject, we determined spike frequency during wakefulness, light NREM sleep (stages 1-2), and deep NREM sleep (stages 3-4), and compared these values to the mean spike frequency during sleep. Spike frequencies were 51-109\% higher in NREM sleep than in REM sleep (Fig. 2). In all subjects, spike counts were lower during REM than during NREM. Spike frequencies were highest during stage 1-2 NREM sleep in 3 subjects and during stage 3-4 sleep in 1 subject (no. 4). We also compared spike frequencies between the 1st and 2 nd halves of the night for each sleep stage and found higher spike frequencies in the 2nd half of the night in all stages of all subjects except for stage REM in subject no. 4 (Table II).

Spike counts varied between 2 electroencephalographers with 1 electroencephalographer reporting $14 \%$ more spikes. Selected pages were reviewed and it was determined that the differences were found to be based on whether or not

TABLE II

Spike frequency by sleep stage.

\begin{tabular}{|c|c|c|c|c|c|c|}
\hline \multirow[t]{2}{*}{ Patient } & \multirow[t]{2}{*}{ Spikes/min } & \multicolumn{5}{|l|}{ Sleep stage } \\
\hline & & Wake & REM & NREM & $1-2$ & $3-4$ \\
\hline \multirow[t]{3}{*}{1} & Total & 0 & $0.18 \pm 0.04$ & $0.30 \pm 0.03$ & $0.44 \pm 0.04^{b}$ & $0.10 \pm 0.02$ \\
\hline & First $1 / 2$ of night & 0 & 0 & $0.13 \pm 0.02^{e}$ & $0.20 \pm 0.04^{\mathrm{e}}$ & $0.09 \pm 0.02$ \\
\hline & Second $1 / 2$ of night & 0 & $0.21 \pm 0.05$ & $0.52 \pm 0.06$ & $0.59 \pm 0.07$ & $0.12 \pm 0.07$ \\
\hline \multirow[t]{3}{*}{2} & Total & $0.06 \pm 0.03$ & $2.7 \pm 0.3^{d}$ & $5.4 \pm 0.2^{a}$ & $6.0 \pm 0.3^{\circ}$ & $4.2 \pm 0.2$ \\
\hline & First $1 / 2$ of night & $0.07 \pm 0.04$ & $1.3 \pm 0.3$ & $3.9 \pm 0.2^{\mathrm{e}}$ & $4.1 \pm 0.3^{\mathrm{e}}$ & $3.5 \pm 0.2^{\mathrm{e}}$ \\
\hline & Second $1 / 2$ of night & 0 & $3.1 \pm 0.3$ & $7.1 \pm 0.4$ & $7.4 \pm 0.4$ & $5.8 \pm 0.6$ \\
\hline \multirow[t]{3}{*}{3} & Total & $29.7 \pm 2.1$ & $28.9 \pm 0.7$ & $43.7 \pm 0.5^{a}$ & $47.5 \pm 0.5^{b}$ & $33.5 \pm 0.9$ \\
\hline & First $1 / 2$ of night & $36.7 \pm 2.6$ & $26.3 \pm 1.0$ & $39.0 \pm 0.4^{e}$ & $46.3 \pm 0.8$ & $30.6 \pm 0.6^{\mathrm{e}}$ \\
\hline & Second $1 / 2$ of night & $14.1 \pm 2.2$ & $31.9 \pm 1.1$ & $48.3 \pm 0.6$ & $48.2 \pm 0.6$ & $49.3 \pm 4.1$ \\
\hline \multirow[t]{3}{*}{4} & Total & $0.91 \pm 0.21$ & $2.2 \pm 0.4$ & $4.6 \pm 0.2^{a}$ & $3.8 \pm 0.2^{b}$ & $6.5 \pm 0.3$ \\
\hline & First $1 / 2$ of night & $1.3 \pm 0.3$ & $2.3 \pm 0.6$ & $4.3 \pm 0.3$ & $3.2 \pm 0.3$ & $6.0 \pm 0.4$ \\
\hline & Second $1 / 2$ of night & $0.21 \pm 0.12$ & $2.1 \pm 0.6$ & $5.0 \pm 0.2$ & $4.2 \pm 0.3$ & $7.6 \pm 0.6$ \\
\hline
\end{tabular}

Mean \pm S.E.M.

${ }^{\mathrm{a}} P<0.0001$ vs. REM and vs. wake.

${ }^{\mathrm{b}} P<0.0001$ vs. stage $3-4$.

${ }^{\circ} P<0.005$ vs. stage $3-4$

d $P<0.0001$ vs. wake.

${ }^{\mathrm{e}} P<0.001$ vs. second $1 / 2$ of night. 
very low amplitude $(10-20 \mu \mathrm{V})$ spikes were counted along with other small sharply contoured transients that were slightly broader than other spikes.

\section{Discussion}

In Rett syndrome, epileptiform activity appears to be maximally expressed during stage 1-2 NREM sleep and in the early morning hours. Epileptiform discharges may decrease with increasing age or increasing disease duration.

Diagnostic criteria for Rett syndrome have recently been formulated (Rett Syndrome Diagnostic Criteria Work Group 1988). Although necessary and exclusionary criteria have been identified, the diagnosis may be tentative for the first few years of symptoms. Rett syndrome is often initially misdiagnosed as autism, a disorder that can be associated with waking apneas (Gastaut et al. 1987). Even in the pseudostationary stage, Rett syndrome may be difficult to distinguish from Lennox-Gastaut syndrome and other static or progressive encephalopathies associated with seizures.

EEG and sleep EEG may help to diagnose patients who are still in the early stages of the disease. Parasagittal short duration spikes which increase in frequency during sleep are characteristic EEG findings (Garofalo et al. 1988), and according to Hagne et al. (1989), these spikes are the first EEG abnormality in $60 \%$ of cases. Such spikes can often be seen on daytime sleep EEG recorded in the EEG laboratory. Our findings indicate that although spike frequency is highly variable, spikes are usually most frequent in the early morning and during light NREM sleep. Although these sleep EEG characteristics are not pathognomonic, they are distinct from the paroxysmal fast spikes and pseudoperiodic slow spike and wave that are characteristic features of the sleep EEG in Lennox-Gastaut syndrome (Beaumanoir 1985; Gabor and Seyal 1986). Rarely, however, EEG features of Lennox-Gastaut syndrome can be seen in patients with Rett syndrome (Olmos Garcia de Alba et al. 1987). Since spikes during sleep EEG appear to be uncommon in autistic children (Dorenbaum et al. 1987), this finding should alert the clinician to the possibility of Rett syndrome.

The reason for increased spike frequency in the early morning hours is uncertain but the increase is not just due to greater amounts of NREM sleep during these hours. Circadian variation in cortical excitability or hormonal factors or both may play a role.

The variation in spike counts between electroencephalographers is not surprising. Interobserver variability in EEG interpretation of specific EEG transients is not uncommon (Williams et al. 1985). In our patients, both electroencephalographers agreed that spikes were present in each recording and the clinical interpretations would have been unaffected by the variation in total spike count.

\section{References}

Beaumanoir, A. The Lennox-Gastaut syndrome. In: J. Roger, C. Dravet, M. Bureau et al. (Eds.), Epileptic Syndromes in Infancy, Childhood and Adolescence. John Libbey, London, 1985: 89-99.

Dorenbaum, D., Mencel, E., Blume, W.T. and Fishman, S. EEG findings and language patterns in autistic children: clinical correlations. Can. J. Psychiat., 1987, 32: 31-34.

Gabor, A.J. and Seyal, M. Effect of sleep on the electrographic manifestations of epilepsy. J. Clin. Neurophysiol., 1986, 3: 23-38.

Garofalo, E.A., Drury, I. and Goldstein, G. EEG abnormalities aid diagnosis of Rett syndrome. Pediat. Neurol., 1988, 4: $350-353$

Gastaut, H., Zifkin, B. and Rufo, M. Compulsive respiratory stereotypes in children with autistic features: polygraphic recording and treatment with fenfluramine. J. Autism Dev. Disord., 1987, 17: 391-406.

Glaze, D.G., Frost, J.D., Zoghbi, H.Y. and Percy, A.K. Rett's syndrome: correlation of electroencephalographic characteristics with clinical staging. Arch. Neurol., 1987, 44: 1053-1056.

Hagberg, B. and Witt-Engerström, I. Rett syndrome: a suggested staging system for describing impairment profile with increasing age towards adolescence. Am. J. Med. Genet., 1986, 24: 47-59.

Hagne, I., Witt-Engerström, I. and Hagberg, B. EEG development in Rett syndrome. A study of 30 cases. Electroenceph. clin. Neurophysiol., 1989, 72: 1-6.

Niedermeyer, E., Rett, A., Renner, H., Murphy, M. and Naidu, S. Rett syndrome and the electroencephalogram. Am. J. Med. Genet., 1986, 24: 195-199. 
Olmos Garcia de Alba, G., Gamboa Marrufo, J.D., Rengifo, Ramos, O. et al. Rett's syndrome with Lennox-Gastaut pattern. Clin. Electroenceph., 1987, 18: 187-190.

Rechtschaffen, A. and Kales, A. A Manual of Standardized Terminology, Techniques, and Scoring System for Sleep Stages of Human Subjects. Brain Information Service/Brain Research Institute, Los Angeles, CA, 1968.

Rett Syndrome Diagnostic Criteria Work Group. Diagnostic criteria for Rett syndrome. Ann. Neurol., 1988, 23: 425-428.
Verma, N.P., Chheda, R.L., Nigro, M.A. and Hart, Z.H. Electroencephalographic findings in Rett syndrome. Electroenceph. clin. Neurophysiol. 1986, 64: 394-401.

Williams, G.W., Lüders, H.O., Brickner, A., Goormastic, M. and Klass, D.W. Interobserver variability in EEG interpretation. Neurology, 1985, 35: 1714-1719. 